\title{
Parler autour du Père mort Les théâtres de paroles de Gérard Bessette et d'Hervé Bouchard
}

\author{
Stéphane INKEL \\ Université Queen's
}

On se souvient du mot de Jacques Ferron qui, cherchant à répondre à l'invitation de la revue La Barre du jour, cénacle de l'écriture formaliste, se définit comme le «dernier d'une tradition orale et le premier de la transposition écrite » (1967, p.27). Cette place, indépendamment de ses nombreux devanciers transcripteurs, décrit en effet au plus près celle qu'a occupée cet écrivain passeur au sein de la Révolution tranquille, lui qui avait ses aises aussi bien chez les écrivains de Parti pris qu'au milieu des conteurs d'une cuisine gaspésienne, là 
même où il a commencé sa carrière de médecin et où il a renoué avec la parole des contes de son enfance.

Prenons maintenant le non moins célèbre incipit des Anthropoïdes : "Nous avançons lentement dans la savane, dit Guito se dit Guito moi Guito je me dis. »(Bessette, 1977, p. 1) Sans insister sur la place stratégique dévolue à la " parolade» dans ce roman aussi ambitieux qu'isolé dans sa jouissance, on peut relever comment se joue le même changement d'épistémè, le même passage d'une parole $a$ priori collective vers le « je » de l'écriture, serait-il divisé, comme chez le dernier Ferron, ou hanté par les nombreuses voix des ancêtres. On sait comment cette place d'intercesseur, Gérard Bessette l'aura incarnée jusque dans son esthétique, que l'on peut aussi bien qualifier de moderne, comme le rappelait Gilles Marcotte, en raison de la force d'attraction déraisonnable qu'il donne au mot, ce trou noir qui aspire toute signification usuelle dans son gouffre, que traditionnelle, en raison cette fois d'un parti pris jamais abandonné pour le roman réaliste (1982, p.15). C'est cette posture d'intercesseur, voire de funambule, qui de son fil suit pas à pas la ligne de fracture d'une société en plein processus de modernisation, que j'aimerais ici convoquer afin de la faire témoigner d'un événement bien précis que Bessette lui-même aura choisi de mettre en scène dans son roman Le Cycle, publié en 1971, en offrant la représentation des effets discursifs de la mort du père. Car ce que le rassemblement d'une famille, sur trois générations, permet peut-être d'évaluer, c'est la nature du profond changement de mentalité alors en pleine effectuation. C'est dans cette perspective qu'il faut sans doute analyser les voix particulières qui cherchent à cerner la place vide du père mort. Des fantasmes de substitution de Gaétane, hésitant entre la brutalité de Monsieur Tardieu et la maîtrise de Mlle Lacoste, 
à celui de fusion collective de Julien, dans l'attente du messie de pacotille Stanislas-Auguste Casavant, c'est surtout l'espace d'un Dieu en plein processus d'évanouissement dans le Québec de la Révolution tranquille que s'applique à cerner leur théâtre de paroles.

On sait comment Bessette faisait de cette question du Père un sujet de préoccupation, cherchant surtout, à travers ces différentes voix du Cycle, à faire entendre celle, intériorisée, de son cadavre, selon une lecture freudienne qu'il faudrait une bonne fois réévaluer ${ }^{1}$. Mais cette préoccupation elle-même en dit long sur la persistance d'une place contre laquelle l'anticléricalisme affirmé de l'auteur ne pouvait rien. Car que fait Bessette en substituant à ce père Barré une galerie de pères tout aussi décevants si ce n'est renforcer une place laissée vacante mais néanmoins toujours opérante dans chacun des monologues $^{2}$ ? La question que je voudrais soulever non pas sur mais à partir du Cycle concerne cet espace laissé vacant par la chute aussi soudaine qu'irrévocable du catholicisme. Se peut-il que sa mémoire ne cesse de hanter un roman qui

\footnotetext{
1 Voir "Gérard Bessette et la recherche du Père ", entretien accordé à la revue Voix et Images, vol. 1, no 3 , avril 1976, p. 322 , p. 324 et p. 326 , où Bessette met en relation les problèmes de la voix, du Père et ce qu'il appelle le «sur-moi collectif ». Il y révèle également son projet initial de faire entendre le monologue du père, à l'image, faut-il le souligner, du monologue d'Addie Bundren dans As I lay dying. La question qui mériterait certes d'être posée, concernerait les raisons de l'absence de ce monologue. On pourrait alors prendre en considération la différence entre faire parler la mère, disons la langue de la mère, langue indéfiniment en puissance, et le père, problème beaucoup plus complexe qui implique d'abord une opération de reconnaissance, comme l'exemple d'Hamlet le prouve aisément.

2 Jurate Kaminska a déjà souligné, à propos du Cycle, comment l'absence du père ne fait que traduire la fonction nécessairement défaillante de ce même père. D'où la recherche effrénée de substituts paternels conduite par l'ensemble des personnages dans leur monologue (1982, p. 196).
} 
cherche pourtant à en circonscrire la perte? On n'a qu'à penser à la place de messie, conspué ou attendu, qu'occupe Julien pour à peu près tous les personnages du roman, pour s'apercevoir comment cette question demeure étroitement liée à celle de la filiation qui apparaît partout en filigrane. Quelle fonction occupe cette figure et quel enseignement nous transmet-elle à propos de ce moment privilégié de l'histoire? Qu'en est-il de cette mémoire aujourd'hui? L'hypothèse que j'aimerais soutenir consiste à repérer une forme de Loi, celle de la tradition, en vigueur aussi bien dans le roman de Bessette que dans le discours actuel du Québec. Mais une Loi, pour le dire dans les termes de l'équation à l'œuvre chez Kafka, qui resterait en vigueur tout en étant privée de signification ${ }^{3}$. Car cette place du Père, pour être demeurée vacante depuis la Révolution tranquille, n'en irradie pas moins dans le moindre discours tentant d'en circonscrire la perte. Voilà en somme ce que je voudrais cerner en relisant Le Cycle, de même qu'un second roman, tout à fait contemporain, celui-là, s'ouvrant lui aussi sur la mort du père et la scène funéraire rassemblant mère et fils autour de son absence, Parents et amis sont invités à y assister, deuxième roman de l'écrivain le plus intéressant, et de loin, de sa génération, Hervé Bouchard.

Retenons donc la question : qu'en est-il de la mémoire du catholicisme aujourd'hui; retenons la scène, celle du père mort autour de qui mère, fils et filles se rassemblent pour chanter; retenons surtout le temps de la question, cette absence

\footnotetext{
3 Je fais ici référence à l'interprétation du grand historien de la Cabbale, Gershom Scholem, telle qu'on la retrouve disséminée dans sa correspondance avec Walter Benjamin. Pour la synthèse la plus complète de cette interprétation, après celles de Derrida, Massimo Cacciari ou Stéphane Mosès, voir Giorgio Agamben, 2006.
} 
d'Histoire, selon les dires mêmes de Bessette, qui dans un entretien affirmait qu'il n'y a d'« Histoire véritable [que] quand on se succède de génération en génération » $(1976$, p. 326). On reconnaîtra ce qui dans une telle affirmation fait de l'entreprise de Bessette quelque chose d'exemplaire quant à ce rapport problématique à l'histoire, ce « spectre qui hante la conscience collective et demande vengeance » (1992, p. 147), comme a pu l'écrire Jean Marcel dans une formule qui cristallise la posture des écrivains québécois de sa génération.

\section{Un Père sans qualité}

Prenons la scène d'ouverture du Cycle, telle que dessinée par les paroles du premier récitant, le petit Jacot, dont il n'est pas indifférent qu'il soit le premier: "Grand-papa est là dans la grande boîte brune il ne bouge plus. » (1971, p. 7) À la fois présent et absent, le grand-père attend dans sa boîte qu'on l'emporte et qu' «on ne le voi[e] plus». Voilà donc la mort reconduite d'emblée à son horizon d'absence sans pour autant cesser de renvoyer au (grand) père. Car c'est toute la finalité de ce monologue d'ouverture, sciemment écrit dans une langue d'enfant - c'est-à-dire traversé de multiples discours, des flammes de l'enfer au petit diable qui le tenaille, mais dont aucun ne parvient encore à faire souche -, de chercher à cerner cet espace de l'absence, d'en dessiner les bords, tout en maintenant la relation de cet espace avec la figure du père, quel qu'il soit. Trait non moins remarquable, cette adéquation de l'espace et de l'absence se retrouve ensuite disséminée partout dans le roman, allant jusqu'à inscrire sa petite case rectangulaire en forme de cercueil dans la typographie même de la page en autant de blancs dont le plus éminent est sans 
doute celui, immense, qui suit immédiatement les mots d'Anita venant d'évoquer les «funérailles de papa » (p.173), comme si la figure du père ne pouvait qu'appeler ce blanc cercueil pour en préserver la place. On en retrouve un autre exemple dans le monologue de Julien lorsqu'il s'inquiète de l'effet de son absence dans les gradins, le mot « absence » se voyant non seulement suivi d'un blanc mais de l'image même de l'espace du "salon funéraire [où] autour d'un cercueil de chêne des âmes pieuses s'agenouillent et prient» (p.102). Mais il revient à Roch, figure même ducycle éternellement repris des générations - «Aujourd'hui c'est lui demain ce sera moi fils aîné [...] je culbuterai à mon tour dans le néant» (p.147) d'énoncer avec le plus de clarté ce vacillement épistémologique dont le roman se fait l'écho lorsqu'il décrit comment «le démiurge paternel sombrait avait sombré peu à peu dans le néant l'insignifiance ", n'arrivant plus pour la mère à faire le poids face au «douillet messie catastrophique» (p. 162) et les valeurs nouvelles que va incarner Julien, au diapason « unanimiste 4 » de la société québécoise.

Plusieurs choses méritent ici d'être soulignées. Allons d'abord à l'essentiel : c'est dans cette non reconnaissance de la mère que le père se vide de ses noms, ne pouvant plus barrer quoi que ce soit pour Julien et Gaétane, qui ne cherchent pas sans raison des pères substituts dans le moindre corps un peu redressé, comme ce Stanislas-Auguste Casavant dont les «bras brandis jambes écartés » forment « l'ossature de fer» sous laquelle Julien "frissonne et vibre à l'unisson d'un peuple» (p. 81). Ces nombreux substituts, autant de figures de

${ }^{4}$ Voir les pages $81,83,85,95,96,105$, etc. 
Commandeur de pacotille ${ }^{5}$, ne cessent donc pas de tomber de leur socle au gré des différents monologues du roman, cédant la place à la seule figure pouvant incarner ce désir de la mère : non pas le Père, mais le fils, dans le roman incarné par Julien, cet « enfantelet transcendant, aux dires de Vitaline, dont les vagissements apaisaient réanimaient dans l'outre-tombe une immense et fantomatique figure » (p.55) et dont la perte symbolique assène sur ses épaules «le poids écrasant de l'impossible rédemption ».

Le deuxième point concerne la fonction occupée par le jugement de Roch sur la déchéance du « démiurge paternel ». Il est remarquable de noter comment, occupé à railler le catholicisme, dont la mort du père ne ferait qu'illustrer la perte d'efficience sur la société québécoise, Roch ne peut le faire qu'en recourant à la parodie, c'est-à-dire au registre même du catholicisme, serait-il dévalorisé, qu'il prétend destituer. Ce faisant, son monologue participe directement, dans l'économie du roman, à l'inversion, nécessairement temporelle, du père et du fils au fondement de toute logique messianique, entérinant en fait, sinon en droit, cette inversion amorcée par la mère. Comment s'y prend-il pour mener à bien cette destitution? Il semble bien, et prenons garde à ce point puisqu'il en va du sens de ce « cycle » donnant le titre au roman, que Bessette cherche ici à opposer le temps cyclique de l'histoire naturelle à l'histoire linéaire du christianisme, selon un nietzschéisme syncrétique qu'il faudrait soumettre à l'examen. Mais le point culminant de cette intervention de Roch consiste évidemment dans ce recours au «néant», qui conduit Bessette à expliciter

\footnotetext{
5 Voir en particulier le «masque impassible et granitique » prêté à Casavant par Julien, p. 88.
} 
l'adéquation toujours opératoire pour l'un ou l'autre termes du nihilisme et du messianisme. Que le «démiurge paternel » sombre dans le «néant» ne disqualifie que lui-même en tant que Père (ou la religion du Père), nullement la place laissée vacante qui devient au contraire l'enjeu de tout ce mouvement de dévalorisation des «valeurs supérieures » qui ont fait l'objet de tant d'analyses de la part de Nietzsche ${ }^{6}$. Mais le fils appelé à occuper cette place, ce "douillet messie catastrophique», comme l'appelle Roch en toute rigueur, ne vaut ainsi qu'à incarner ce pur néant de la Loi, c'est-à-dire à remplir son rôle de messie visant à suspendre, en l'accomplissant, la fonction de la Loi7. Voilà donc les différents enjeux que cette représentation de la mort du père permet de mettre au jour dans le romanbilan de Bessette. Une absence qui se décline sur le mode de la destitution, si ce n'est de l'imposture, et qui loin d'abolir la fonction (la place) du Père, ne s'attaque qu'à sa figure (à son masque). Ce qui laisse indéfiniment maintenue la puissance d'une Loi qui, si elle ne signifie plus rien, peut toujours faire retour dans les méandres de l'histoire.

\section{"Le temps nul d'un commencement fini »}

Qu'en est-il de cette place aujourd'hui, demandions-nous en ouverture? A-t-on raison de craindre un retour du refoulé en ce

\footnotetext{
6 Voir en particulier La généalogie de la morale, in OEuvres philosophiques complètes, t. VII, éd. Colli-Montinari, Gallimard, 1971, de même que l'analyse de cette «place » par Heidegger dans « Le mot de Nietzsche " Dieu est mort " » inChemins qui ne mènent nulle part, Gallimard, coll. « Tel », 1996, p. 308.

7 Voir Giorgio Agamben, qui commente cette adéquation du messianisme et du nihilisme en montrant comment le messianisme « réduit à rien la Loi mais en maintient le rien dans une "vigueur" perpétuelle et infiniment différée» (2006, p. 225).
} 
qui concerne la tradition du catholicisme si aisément rejetée? Avec Hervé Bouchard, dans Parents et amis sont invités à $y$ assister (2006), nous retrouvons la même scène d'un père autour duquel se pressent « parents et amis » plus de trente ans après la parution du roman de Bessette. Un roman à l'esthétique radicalement différente, il va sans dire, si ce n'est un même découpage en "paroles", dans le cas du roman de Bouchard directement calqué sur la forme du théâtre. Si l'on retrouve dans ce roman la même fonction attachée au dernierné de la veuve Manchée, il faut immédiatement noter comment l'espace de la mort du père n'est pas du tout délimité mais au contraire disséminé, dans ce théâtre de paroles fondé sur une logique du performatif8. Une figure disséminée en autant de signifiants et de figures théâtrales à la parodie assumée, dont la moindre n'est pas « l'épisodique Laurent Sauvé » pour laquelle le théâtre se doit d'engager, comme le spécifie la didascalie initiale, s'il a des moyens, un «fils de dieu pour se tenir derrière $^{9}$ » (p. 12). Mais ce qu'il est important de retenir, c'est l'effet de rupture que provoque la mort du père et qui entraîne cette dissémination qui se voit directement reliée au trou non pas dans l'espace mais dans le temps des orphelins, comme le résume l'orphelin de père numéro deux :

Ç’a été, non. Ç’a été, non ça n'a pas été. Je ne sais pas ce qui a été. Ç’a été impossible de parler. Et pourtant tout le monde on

8 Cette logique est définie d'entrée de jeu par une didascalie qui en forme l'ouverture: "Noir dans le théâtre où la scène est crayonnée. Quand les persons (sic) parlent, ils disent et on voit. L'un [...] dit la veuve Manchée et elle est. Le mari Beaumont, il le dit couché mort et il est couché mort au centre. Il dit une suite de six qui sont orphelins de père et ils sont [...] On entre dans l'art tisonné de démonstrations hurlées où énoncer signifie produire le théâtre où la scène est crayonnée. » (p. 11-12)

9 Voir aussi la suite de l'énoncé : «Sinon, un ressuscité quelconque fera l'affaire. » 
s'est senti en nuée pleine de paroles, la lumière a comme viré [...], et comme elle virait les mots qu'on avait cru connaître se sont mis à flotter en groupes, ça nous les rendait étrangers, pas moyen d'en prononcer un, ç'a été impossible de continuer à être [...] (p. 32)

On le voit, ce que cette impossibilité d'ordre ontologique vise surtout à souligner, c'est un changement de qualité affectant la nature même du temps des orphelins, dès lors défini en tant que «temps nul d'un commencement fini » (ibid.). Mais que signifie donc un tel temps? Qu'en est-il de cette valeur de néant accolée non pas au "Père » démiurgique mais au temps lui-même? Là réside toute la force du roman de Bouchard et son importance à la fois pour l'histoire littéraire et pour l'histoire des idées, au Québec, dans laquelle il provoque un bouleversement épistémologique qu'il s'agit de voir et de prendre en compte : ce qui à première vue semble un accroissement du nihilisme s'avère au contraire une toute nouvelle manière, rigoureusement conséquente, de mettre en question le temps de l'histoire suite au dépérissement de l'autorité affectant la tradition (catholique) - ou, si l'on veut, une manière de composer avec la mise en suspens de la Loi. La mort du père agit ainsi comme un aiguillage qui propulse les orphelins dans une voie parallèle où la temporalité et les mots censés en rendre compte ne correspondent plus à leur image usuelle. Un changement de qualité qui affecte donc aussi bien le temps que le rapport à la langue. Un espace est ouvert par la mort et il engouffre tout, y compris les mots des orphelins qui ne parviennent plus à le cerner.

On peut ainsi noter une première disjonction entre le roman de Bessette et celui de Bouchard dans la fonction qu'ils attribuent à cette mort du père qui leur sert de prétexte. Alors 
que le premier appelle à la barre ce " Père primordial ", comme il le nomme, afin d'en faire le procès, montrant surtout la prédominance d'un fils qui lui-même, nouveau Stephen Dedalus, ne peut que partir à la recherche d'un père substitut, le second dès l'ouverture rompt le cycle, fait de la mort du père Beaumont non pas un événement parmi d'autres, serait-il lourd de modifications quant à l'autorité de la Loi, mais l'avènement eschatologique d'un temps en plein processus de transformation, temps de la récapitulation de «l'intégralité du passé $^{10} »$ dans la figure du messie, pour le dire avec Paul, que résume bien l'orphelin de père numéro un lorsqu'il affirme sa crainte que «le présent [soit] fini, [...] qu'à l'avenir il n'y [ait] plus que le passé qui nous ferait» (p. 33). Tout se passe comme si le choix délibéré de mettre en scène la mort du père était aussi pour Hervé Bouchard, comme pour Bessette, un prétexte afin de tenter de représenter un évanouissement beaucoup plus marquant, celui de l'autorité d'un objet de croyance qui n'en demeure pas moins tapi dans l'ombre de la mémoire et qui revient hanter une conscience historique en mal de figures de remplacement. Mais une représentation qui serait à la hauteur de cet avènement épistémologique, ce qui implique un autre rapport au temps et de toutes nouvelles figures afin d'en rendre compte ${ }^{11}$.

10 Voir Giorgio Agamben (2000, p. 125), qui commente Eph., 1, 10 : «Pour l'économie du plérôme des temps, toutes les choses se récapitulent dans le messie, aussi bien les choses célestes que les choses terrestres. »

11 On reconnaîtra ici une terminologie empruntée au maître livre de Fernand Dumont, Le Lieu de l'homme, qui décrivait sous le registre de l'avènement la « crise du langage » propre à la modernité, décrite avec l'aide de ses œuvres les plus marquantes (Flaubert, Mallarmé, Blanchot), qui vise précisément à « récuser » l'histoire (1994, p. 102, sqq.). C'est aujourd'hui une évidence de constater à quel point la rupture de la Révolution tranquille, sur laquelle il n'aura de cesse de s'interroger à partir des années 1980, transparaît dans 
Il faudra donc également un fils "attendu», un autre «chouchou messianique», comme le dit Roch à propos de Julien, pour bien marquer le rôle de la mère dans cette affaire de filiation et de désir; mais cette fois, en toute logique, parce qu'il est appelé par cette modification qui affecte le temps, plus que jamais temps de la mémoire à la recherche d'une figure qui en ferait la somme. À ce «temps nul d'un commencement fini » de l'orphelin de père numéro deux, forme poétique de cette «antichambre de l'historicité » dont parlait Jean Marcel à propos de Saint-Denys Garneau (p. 152), doit correspondre une figure de l'attente qui ne cherchera pas tant à rouvrir ce temps clos de l'histoire qu'à en offrir une image condensée, une image dialectique pour le dire avec Benjamin ${ }^{12}$, capable d'en faire l'interprétation, c'est-à-dire d'en saisir les effets afin de rendre au présent sa pleine lisibilité.

\section{Quel messie pour le temps de maintenant}

Cette figure de "l'attendu», dans Le Cycle aussi bien que dans Parents et amis sont invités à y assister, prendra évidemment les traits de celle du messie. Figure polymorphe par excellence, le messie est en effet une forme vide, signifié chargé de sens jusqu'à exploser mais à la plasticité

chacune des pages de cet ouvrage exclusivement théorique où le Québec n'est jamais directement mentionné, si ce n'est par allusion dans les dernières pages du chapitre 6, consacré à «La culture en tant que conscience historique ».

12 « Chaque présent est déterminé par les images qui sont synchrones avec lui; chaque Maintenant est le Maintenant d'une connaissabilité déterminée [...]. Il ne faut pas dire que le passé éclaire le présent ou que le présent éclaire le passé. Une image, au contraire, est ce en quoi l'Autrefois rencontre le Maintenant dans un éclair pour former une constellation. En d'autres termes, l'image est la dialectique à l'arrêt. » (2000a, p. 479) 
étonnamment malléable, indistincte, pouvant toujours être saisi par qui voudrait en exploiter les potentialités révolutionnaires ${ }^{13}$. Gérard Bensussan a bien montré comment «le messie n'est pas un homme, c'est un temps, voire la temporalité du temps » (p. 46). Figure titulaire de l'attente, le messie est surtout celui qui en transforme totalement l'expérience dans la mesure où il est chargé de faire coïncider l'histoire avec le sort de ceux qui en auraient été exclus. Mais en tant qu'attendu, précisément, comme le texte évangélique en a si bien saisi les contours, le messie a tout à voir avec le désir et se confond de fait avec la figure de la filiation. Et c'est bien ce qui étonne, dans ces deux romans, d'avoir fait de la filiation leur question centrale au détriment de la question du père, malgré la position prééminente qu'elle occupe de prime abord dans l'un et l'autre. Comme si c'était en effet le fils, la parole du fils, qui « engendre le père », pour citer le mot du Saint-Élias de Jacques Ferron ${ }^{14}$, dans un bouleversement chronologique qui dit bien, il me semble, la place prépondérante de l'énonciation pour qui cherche à se colleter avec un héritage qui n'a toujours pas de place identifiable au sein de la mémoire d'où il serait possible de la convoquer. Si pour le Québec « l'histoire est un spectre ${ }^{15}$ »,

\footnotetext{
13 On se référera ici aux travaux de Gershom Scholem, qui font toujours autorité, en particulier à son étude exhaustive portant sur le « messie » du XVIIe siècle qui a bouleversé l'univers des ghettos, Sabbataï Tsevi. Le messie mystique, Paris, Verdier, « Les Dix Paroles », 1983.

${ }^{14}$ Locution centrale du roman, d'ailleurs répétée à trois reprises $(1993$, p. 54, 99, 149).

15 Marcel, p. 147. Une affirmation qui est ici à mettre en rapport avec la célèbre affirmation de Stephen Dedalus, surtout quand on sait la place qu'occupe le spectre d'Hamlet à l'intérieur de Ulysses: «History is a nightmare from which I am trying to awake » (James Joyce, Ulysses, Annotated Student Edition, New York, Penguin books, [1922] 1992, p. 42). C'est à ce parallèle entre l'Irlande et le Québec, dans leurs rapports respectifs à l'histoire, que Victor-Lévy Beaulieu a consacré son dernier grand ouvrage,
} 
à conjurer, donc, le « Père primordial » est d'autant plus difficile à conjurer qu'il est évanescent, infigurable dans son exil, d'où la difficulté de faire son histoire et d'ainsi procéder à ce que Michel de Certeau, dans L'écriture de l'histoire, appelle son « rite d'enterrement», c'est-à-dire marquer un passé, "faire une place au mort [afin de] redistribuer l'espace des possibles » (1975, p. 118). Il est à cet égard intéressant de constater comment le roman qui suit immédiatement Le Cycle, mais après plusieurs années consacrées à sa difficile gestation, met en scène de manière symptomatique la lente advenue de la " parolade » pour un autre « fils » à la paternité incertaine ${ }^{16}$. Le « rite d'enterrement» auquel on assiste, dans Le Cycle, restera donc incomplet, faute de dépouille. Quant au roman de Bouchard, il ne nous convoque pas tant à un enterrement qu'à une cérémonie où l'on s'applique à disperser les cendres du défunt. Il ne reste plus que poussières de mots dispersés dans les différents discours qui n'aspirent plus à dire ni le père ni le catholicisme qui se cache derrière. Tout au plus butera-t-on sur la lettre «J », ou sur le « grand J », dans un sermon parodique à propos de la figure de Lazare, le ressuscité, cela en toute rigueur avec cette logique de la lettre que le roman a cherché à

après plusieurs années de préparation. Ouvrage d'autant plus intéressant qu'il s'agit du retour du personnage d'Abel. Voir James Joyce, l'Irlande, le Québec, les mots, Trois-Pistoles, Éditions Trois-Pistoles, 2006.

16 Agnès Whitfield, insistant plutôt sur la remise en question du modèle patriarcal dans Les Anthropoïdes, a bien montré comment le jeune Guito multiplie les pères symboliques dans sa recherche d'une parole fondatrice, de « Klabou-à-la-langue-vipérine » à « Salaloudi-le-disert » (1991, p. 48-51). Il faudrait quant à cette intrication du père et de la parole fondatrice entreprendre une étude comparée entre la tentative bessettienne des Anthropoïdes et le Monsieur Melville de Victor-Lévy Beaulieu, publié tout juste un an plus tard. La différence tiendrait sans doute dans cette volonté de conciliation que l'on retrouve chez Bessette, rejetée avec détermination chez Beaulieu. 
mettre en place. Les deux romans insistent donc sur la figure du fils, ou des fils, pour être exact, plutôt que sur celle du Père impossible à circonscrire. C'est à l'analyse de cette figure que nous consacrerons par conséquent nos derniers développements.

Premier constat : cet accent mis sur la filiation, dans les romans de Bessette et de Bouchard, ne les empêche pas de mettre tous deux en scène ce qu'on peut appeler la recherche forcenée d'un père impossible. On sait comment Julien va chercher, dans un processus d'identification inachevé, à lier ses « ancêtres humiliés » (1971, p. 81) au chant « unanime» de la collectivité, désormais «sans chapelet sans famille [où il] élabore un collectif avenir [pour venger] tout un peuple» (p. 96). Mais cet avenir est lui-même ce qui fait problème, dans ce roman, comme le montre bien la temporalité cyclique du monologue de Roch, confirmée par une nouvelle recherche du Père dans le monologue d'Anita. Avec l'orphelin de père numéro six, lui aussi en mal de figures paternelles, le roman de Bouchard se confronte avec la même mélancolie du fils, à la différence notable qu'il s'agira ici de l'approfondir jusqu'à sadéfiguration afin d'œuvrer au plus près de cette potentialité polymorphe du «messie» ouvert à tous les temps. C'est à présenter ce rôle qui ne lui revient pas, malgré sa tête de " ressuscité », que va servir l'épisodique Laurent Sauvé, puisque c'est lui qui va attirer l'attention des autres orphelins sur la « joie » cachée par la robe en bois de la veuve Manchée : « elle a le corps là mais elle entend de toutes parts les voix outrées et les chants obscurs de la viande en elle qui lui parfume le trou. Elle est visitée, regardez, regardez. » (2006, p. 41) C'est à même ce drame "du six», après l'ellipse nécessaire à son entrée en 
langue ${ }^{17}$, qu'il est possible d'apercevoir cette logique de l'effacement qui affecte la figure de celui qui se désigne du nom d' «attendu » et d' " espéré », lui qui progressivement se videra aussi bien de son nom que de cette fonction d'ouvreur d'avenir.

Il commencera ainsi, lui aussi, par multiplier les pères dont il établira la liste, qu'il murmurait «en vrai [Hamlet] obsédé de l'origine » (p. 177), comme si le manque qui préside à son entrée dans le symbolique ne pouvait se résoudre que par l'effacement de sa propre identité. Il perdra ainsi logiquement son nom pour devenir ce "nombre » qui parle à la suite des autres, avant de rouler à vélo dans la mort « à la recherche de lui » (p. 145). C'est à la fin de son chant de suicidé qu'il se qualifiera lui-même de toute une série de noms qu'il nous importe grandement de relever puisque c'est en eux que se rassemble la logique temporelle de cet avènement que nous avons cherché à cerner :

Je suis celui demain qui sera, je suis celui qu'on retrouvera, je suis celui dont on dira qu'il eut pour pères tout un troupeau, je suis celui dont on cherchera la tête de veau, je suis le bras aimant de ma mère, je suis l'étrangleur au fil blanc, je suis le dernier né du premier jour de l'an prochain, je suis l'attendu, l'espéré, le fêté de fin juin, le noyé de fin mai, le découvert d'avril, le tombé de mars, ça devrait aller comme ça. Ça devrait aller comme ça. (p. 149)

On peut se demander ce que signifie cette suite de noms qui subitement recouvrent celui qui n'en avait plus, ce collier de « je suis » autour de celui qui affirmait ne pas pouvoir être. C'est que la mort du six le révèle subitement à cette fonction dont les autres orphelins l'avaient d'emblée recouvert, l'effacement de

17 Voir la didascalie de p. 45 : «Qu'il grandisse, mais pas trop. Amenez-le au seuil de la parole puis abandonnez-le, il chantera.» 
sa propre identité n'étant que le symptôme d'une indétermination bien plus signifiante. Car c'est par cette suite de noms et le bouleversement temporel qui l'accompagne que l'orphelin de père numéro six rejoint la figure exemplaire du messie recouvert de tous les noms de l'histoire. Avec cette figure creuse comme un signifiant, Hervé Bouchard propose par conséquent l'équivalent en fils du personnage joycien de H.C.E., ce Here Comes Everybody à l'invite du lecteur, appelant ainsi implicitement à reconnaître en lui le passé à commémorer afin de passer à l'avenir, seule logique de l'histoire, comme l'écrit Benjamin à la fin de ses thèses "Sur le concept d'histoire », où « chaque seconde [est] la porte étroite par laquelle le Messie [peut] entrer» (2000b, p. 443). Alors pourquoi ce registre messianique de «l'attendu »? Précisément parce que le messie sert ici de figure de contraction, de récapitulation d'une histoire qui au Québec s'est partagée selon une double détermination : problématique, d'abord, comme le montre la route poursuivie par le «peuple sans histoire » de Durham dans l'écriture de la Révolution tranquille, de Miron à Hubert Aquin, et religieuse, c'est-à-dire providentielle, c'est-à-dire promise. Mais les deux allaient évidemment de pair, le registre providentiel ne faisant qu'épouser la logique d'une histoire dont l'entrée se voyait constamment repoussée à plus tard. C'est à réinterpréter cette logique du registre messianique selon les termes d'une reconnaissance, et d'une assomption de l'entièreté du passé qu'il faut désormais s'attaquer si l'on veut sortir des impasses d'une historicité qui au Québec peine toujours à se déployer.

Qu'en est-il de la question? Je dirai que si je me suis attardé si longuement à ce roman de Bouchard, c'est qu'il me semblait 
reprendre ce projet du Cycle de faire le point, par le topos de la mort du Père, sur la disparition de l'horizon religieux au Québec. Mais plutôt que d'en cerner la place pour insister sur une absence, vécue dans Le Cycle sur le mode d'une joie libérante, pour le dire avec Borduas, qu'il ne nous appartient d'ailleurs nullement, nous des générations subséquentes, de juger, le roman de Bouchard s'est appliqué à disséminer les signifiants d'un espace symbolique au cœur de la référence québécoise - comme a pu le montrer Fernand Dumont dans sa reconstitution patiente de la Genèse de la société québécoise. Un théâtre de paroles hanté par ces signifiants, mais dont la présence diffuse forme peut-être la stratégie la plus sûre afin de se préserver d'un retour du refoulé dont la charge explosive serait à l'égal du poids de cette expérience du religieux dans l'histoire. Quant au temps de la question, elle était déjà comprise dans la prose même de Gérard Bessette, qui recueillit au sein même de sa phrase ce rapport au temps et à l'histoire si caractéristique du Québec. Il n'y a qu'à ouvrir n'importe lequel de ses romans depuis L'Incubation pour se heurter à ces phrases qui ne finissent plus, et surtout à cet enchaînement si particulier de verbes agglutinés les uns sur les autres comme pour cerner au plus près l'objet d'une description ou le plus souvent l'émotion du monologuiste. Mais cette suite de verbes ou d'adjectifs, qui se jouent de toutes les règles de la prosodie, ne fait que s'engouffrer dans cette pointe d'éternité qui résulte de cette position d'extériorité à l'égard de l'histoire. Bessette aurait sans doute fait sienne ce propos du personnage du roman de Bouchard, qui affirme que «ce n'est pas soi qu'on révèle en contant sa propre histoire, c'est le temps qu'on perce » (2006, p. 226). Un bouleversement du temps qui donne à l'écriture une liberté certaine, ce qui ne l'empêche pas 
de jeter de temps à autre un regard inquiet vers l'avant, en attente de «l'événement [qui] aura lieu quand il aura lieu c'està-dire tantôt c'est-à-dire dans huit c'est-à-dire demain » (2006, p. 140).

\section{Bibliographie}

AgAmBen, Giorgio (2006), «Le Messie et le souverain. Le problème de la loi chez Walter Benjamin », dans La Puissance de la pensée. Essais et conférences, Paris, Bibliothèque Rivages, p. 213-229.

- (2000), Le Temps qui reste, Bibliothèque Rivages.

Benjamin, Walter (2000a), Paris, capitale du XIXe siècle. Le livre des Passages, Paris, Cerf, coll. « Passages ».

- (2000b), "Sur le concept d'histoire», dans CEuvres III, Paris, Gallimard, coll. « Folio essais », p. 427-443.

BENSUSSAN, Gérard (2001), Le temps messianique. Temps historique et temps vécu, Paris, Vrin, coll. "Problèmes \& Controverses ».

BessetTE, Gérard (1971), Le Cycle, Montréal, Éditions du jour, coll. « Romancier du jour ».

- (1977), Les Anthropoïdes, Montréal, La Presse.

BOUCHARD, Hervé (2006), Parents et amis sont invités à y assister, Montréal, Le Quartanier.

Certeau, Michel de (1975), L'Écriture de l'histoire, Paris, Gallimard, coll. « Bibliothèque des histoires ». 
Dumont, Fernand (1994 [1968]), Le Lieu de l'homme. La culture comme distance et mémoire, Montréal, Bibliothèque québécoise.

FERron, Jacques (1993 [1972]), Le Saint-Élias, Montréal, Typo.

FiSETTE, Jean (1976), « Gérard Bessette et la recherche du Père entrevue », Voix et Images, vol. 1, no 3, p. 317-328.

KAMINSKA, Jurate (1982), «Le fonctionnement de l'espace dans Le Cycle » dans J.-J. Hamm (dir.), Lectures de Gérard Bessette, Montréal, Québec / Amérique, coll. «Littérature d'Amérique / essai », p. 189-197.

MARCEL, Jean (1992), «Écriture et histoire» dans Pensées, passions et proses, Montréal, L'hexagone, coll. "Essais littéraires », p. 143-156.

MARCOTTE, Gilles (1982), «Words, words, words» dans J.-J. Hamm (dir.), Lectures de Gérard Bessette, Montréal, Québec / Amérique, coll. "Littérature d'Amérique / essai », p. 11-18.

Whitfield, Agnès (1991), "Gérard Bessette, écrivain : à la recherche de l'homme nouveau ", Queen's Quarterly, vol. 98, no 1 , p. 40-57.

\section{Résumé}

À trente ans d'intervalle, Le cycle de Gérard Bessette (1971) et Parents et amis sont invités à y assister d'Hervé Bouchard (2006) visent tous deux à mettre en scène les effets de la mort du père sur ceux qui en subissent le deuil tout en insistant sur la modification de leur mode d'appréhension de la temporalité. Si l'écriture de l'histoire s'apparente, suivant Michel de Certeau, 
à un « rite d'enterrement » qui consiste à « faire une place au mort [pour] redistribuer l'espace des possibles ", il s'agira de voir comment ce processus s'avère problématique dans $L e$ cycle en raison d'un déficit symbolique de la figure du père qui reste à interroger. Car ce qui étonne, à la lecture de ces deux romans, c'est précisément d'avoir fait de la filiation leur question centrale au détriment du père dont l'écriture se proposait pourtant de cerner l'absence. On cherchera à interroger le sens du registre de l'attente qui s'inscrit par le biais de la figure récurrente du "messie » accolée à ces fils privilégiés que sont Julien et l'orphelin de père numéro six voyant dans l'un et l'autre romans le symptôme d'une rupture épistémologique dans la représentation romanesque de l'historicité.

\begin{abstract}
Although more than thirty years separate their dates of publication, Le cycle by Gérard Bessette (1971) and Parents et amis sont invités à y assister by Hervé Bouchard (2006) both aim to depict the effects of a father's death on the surviving family. In the process, the novels also highlight the resulting modification of the characters' way of perceiving temporality. If the writing of history is similar to a "burial rite", as Michel de Certeau puts it, it will be shown how this process is difficult within Le cycle because of the symbolic deficit of the father's figure, an element that calls for further study. In fact, what is most surprising in both these novels is precisely how they insist on filiation rather than the explicit topic of the father. This article will thus examine the significance of the
\end{abstract}


expectation (l'attente) that arises with the figure of the "messiah" that is linked to the favourite sons, Julien and the orphan son number six, questioning the epistemological rift of historicity depicted in both novels. 